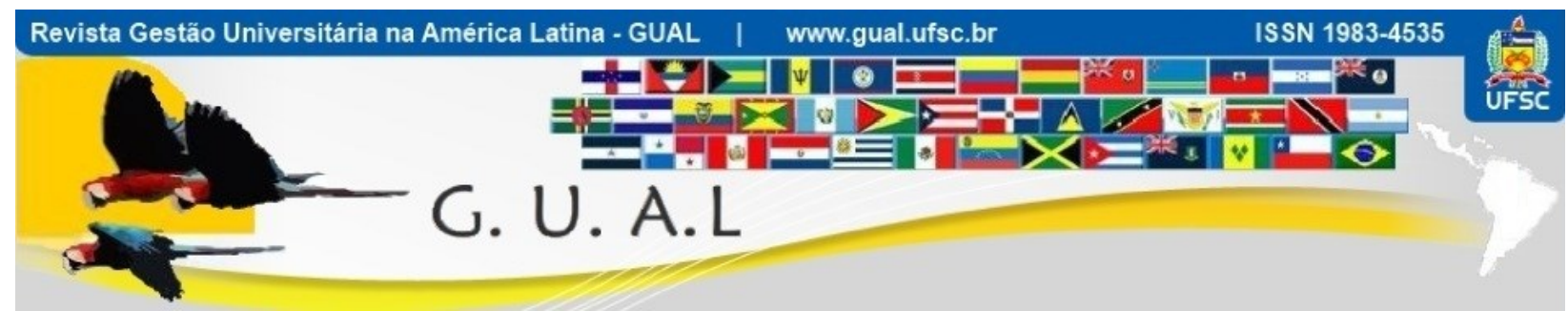

DOI: http://dx.doi.org/10.5007/1983-4535.2017v10n3p1

\title{
ESCALA DE CONTRATO PSICOLÓGICO DOCENTE: ESTRUTURA E EVIDÊNCIAS DE VALIDAÇÃO
}

\author{
ACADEMIC PSYCHOLOGICAL CONTRACT SCALE: STRUCTURE AND \\ EVIDENCE OF VALIDITY
}

\author{
Mino Correia Rios, Doutor \\ Universidade Federal da Bahia - UFBA \\ Unidade Lauro de Freitas - UNIME \\ mino.rios@gmail.com \\ Sônia Maria Guedes Gondim, Doutora \\ Universidade Federal da Bahia - UFBA \\ sggondim@gmail.com
}

Recebido em 23/fevereiro/2016

Aprovado em 14/junho/2017

Sistema de Avaliação: Double Blind Review 


\title{
RESUMO
}

O estudo apresenta uma escala de contrato psicológico para o contexto dos docentes do ensino superior e evidências de validação. Utilizou-se uma amostra de 232 docentes de diferentes regiões do Brasil, de ambos os sexos e com idades variando de 25 a 70 anos. A escala foi construída com base no modelo de trocas sociais homomórficas e heteromórficas, tendo os seus itens construídos a partir de entrevistas com docentes e coordenadores de curso. Os resultados indicaram adequação do modelo, com a validade confirmatória assumida tanto para as obrigações docentes $\left(\chi^{2} / \mathrm{gl}=2,520 ; \mathrm{GFI}=0,859 ; \mathrm{AGFI}=0,854 ; \mathrm{RMSEA}=0,065\right)$, quanto para as contrapartidas institucionais $\left(\chi^{2} / \mathrm{gl}=2,520\right.$; $\mathrm{GFI}=0,859$; $\mathrm{AGFI}=0,854$; RMSEA $\left.=0,065\right)$. Além disso, a medida também aponta bons índices de confiabilidade.

Palavras chave: Contrato psicológico; Escala; Docentes de Ensino superior.

\begin{abstract}
This study aimed to develop and validate a psychological contract measurement for university and college teachers. It was used a sample of 232 teachers from different regions of Brazil, of both sexes and ages ranging from 25 to 70 years. The instrument was developed based on homomorphic and heteromorphic exchanges model, and its items were based on interviews with teachers and coordinators. Results indicated adequacy of the model, with the confirmatory validity assumed for both, teachers obligations $\left(\chi^{2} / \mathrm{df}=2,520\right.$; GFI $=0.859$, AGFI $=0.854$, RMSEA $=0.065)$, and institutional compensations $\left(\chi^{2} / \mathrm{df}=2,520 ; \mathrm{GFI}=\right.$ 0.859 , AGFI $=0.854$, RMSEA $=0.065)$. Furthermore, the measurement also shows good reliability indexes. These data indicate that the instrument is promising, whether in the context of research in higher education, whether in management education.
\end{abstract}

Keywords: Psychological contract; Validation; Teachers, Higher Education 


\section{INTRODUÇÃO}

O período que se inicia na segunda metade do século XX se caracteriza pelo acúmulo, intensificação e aceleração das transformações nas mais diversas esferas da vida humana (e.g., Cooper, 2008). No cenário educacional do país houve mudanças na política nacional de educação, com impactos no aumento expressivo de instituições privadas e públicas, ampliando o quadro de oferta de cursos e vagas de ensino superior e de pós-graduação (e.g., Carvalho, 2006; Franco, 2008; Martins, 2009; Severino, 2008). Uma reconfiguração começou a ser desenhada nas relações entre docentes e suas organizações empregadoras com impactos nos contratos formais e no que se pode denominar contratos psicológicos.

$\mathrm{O}$ conceito de contrato psicológico surge em meados do século $\mathrm{XX}$ e se refere às percepções e expectativas de trocas entre empregado e organização. O contrato formal estabelece direitos e deveres empregatícios com o respaldo da lei, mas não consegue dar conta de todas as expectativas e crenças que cercam a relação de trabalho (e.g., Farnsworth, 1982; Rousseau, 1995). O contrato psicológico contempla esta subjetividade, cujos aspectos perceptivos e afetivos cumprem uma importante função nos vínculos que o trabalhador estabelece com a organização. O trabalhador recém-ingressado ou até mesmo antes, alimenta crenças sobre as obrigações que o empregador deveria ter para com ele, assim como crenças de quais seriam suas próprias obrigações para com o empregador. Neste cenário de potenciais trocas esperadas entre os atores, os vínculos com o trabalho e a organização são construídos, com impactos na qualidade do desempenho do trabalhador e no seu bem-estar (e.g., Bal, Chiaburu, \& Diaz, 2011; Bal, \& Smit, 2012; Hao Zhao, Wayne, Glibkowski, \& Bravo, 2007; Jamil, Raja \& Darr, 2013; Menegon \& Casado, 2006; Wellin, 2007).

Ao ser compreendido como uma "relação de intercâmbio implícita que existe entre um funcionário e a organização", envolvendo "as crenças relativas aos termos e condições de um acordo de trocas recíprocas entre um indivíduo e um grupo" (Rousseau, 1995, p. 19), o contrato psicológico tem seu estudo dividido em duas linhas principais: o conteúdo do contrato psicológico, ou seja, os elementos que estão sustentando a percepção de trocas entre os atores (e.g., Cavanaugh \& Noe, 1999; Chu \& Kuo, 2012; Gammie, 2006; Herriot, Manning \& Kidd, 1997; Janssens, Sels \& Van Den Brande, 2003; Rios \& Gondim, 2010b); e a ruptura do contrato e seus efeitos, cujo foco recai na compreensão do que leva o trabalhador a perceber se houve quebra ou violação de um contrato, com impactos em termos afetivos, motivacionais e de rompimento do vínculo com a organização (e.g., Andersson, 1996; Bao, 
Olso, Parayitam \& Zhao, 2011; Bordia, Restubog \& Tang, 2008; Chin \& Hung, 2013; Conway \& Briner, 2002; Kickul, 2001; Lapalme, Simard \& Tremblay, 2011; Robinson, \& Morrison, 2000).

O estudo dos contratos psicológicos oferece um campo profícuo para o debate em torno da dinâmica nas relações de trabalho e seus efeitos, sobretudo por colocar ênfase na noção de reciprocidade social (e.g., Chiuzi \& Malvezzi, 2014). A temática da reciprocidade como elemento central e de estabilidade nas interações sociais teve contribuição de Gouldner (1960). O estudo desenvolvido por Chiuzi (2014) recupera as ideias desse autor ao destacar que embora as pessoas esperem trocas justas ao longo do tempo e contrapartidas para o investimento feito, a avaliação dessas contrapartidas leva em conta dois aspectos fundamentais: a equivalência em termos de forma e a imediatez em termos de tempo. A reciprocidade homomórfica e a reciprocidade heteromórfica estão relacionadas à equivalência das trocas esperadas. No primeiro caso, espera-se que as trocas sejam equivalentes em termos de conteúdo ou forma. No último caso, ou seja, na reciprocidade heteromórfica, tolera-se ou espera-se que as trocas sejam de natureza distinta. O segundo aspecto da avaliação das trocas é a imediatez, em que a percepção de justiça leva em conta o tempo em que a troca é concretizada. Esta dimensão está mais sujeita a variações individuais, tendo em vista que um dos aspectos centrais da regra de reciprocidade é que quem recebe algo de outra pessoa tornase um "devedor moral", nem sempre se estipulando quando a "dívida moral" será de fato retribuída. Do mesmo modo, quem oferece algo a outrem sustenta a crença de que o recebedor terá uma dívida moral a lhe ser "paga" futuramente. Dada as características das relações de trabalho, as expectativas de imediatez da reciprocidade são mais demarcadas, haja vista que um trabalhador é remunerado por hora ou por mês.

A despeito do crescente número de investigações sobre o tema, o cenário brasileiro é ainda carente em termos de trabalhos sobre contratos psicológicos (e.g., Bastos, Maia, Rodrigues, Macambira, \& Borges-Andrade, 2014; Rios \& Gondim, 2010a). Em se tratando especificamente dos contratos psicológicos de docentes, o número de investigações é ainda mais reduzido, sendo igualmente raras no contexto internacional (e.g., Amparo Sobrinho, 2011; Dabos \& Rousseau, 2004; Krivokapic-Skoko \& O’Neill, 2008; Schen, 2010; Tipples, Krivokapic-Skoko, \& O’Neill, 2007; Wade-Benzoni, Rousseau, \& Li, 2006).

O trabalho docente de ensino superior vem crescendo em complexidade tendo em vista um conjunto de políticas públicas adotadas nos últimos anos pelos governos federais. Além disso, houve uma descoberta de um nicho de mercado para a oferta de serviços educacionais 
de ensino superior presencial e à distância e de pós-graduação lato e de stricto sensu (e.g., Carvalho, 2006; Laus \& Morosini, 2005; Martins, 2009; Nunes, 2012; Stallivieri, 2007). Certamente este cenário tem impactos nos contratos formais de docentes com instituições educacionais empregadoras, e também nos contratos psicológicos. Dois eixos centrais estão na base do exercício do ensino superior independentemente do vínculo institucional público ou privado que venha a ter o docente. O primeiro é o eixo acadêmico e diz respeito tanto à dedicação à formação, ou seja, ao processo ensino-aprendizagem, quanto à pesquisa, isto é, ao processo de produção do conhecimento. É importante salientar, no entanto, que nem toda instituição de ensino superior exige ou proporciona ao docente a oportunidade de se dedicar à pesquisa, estando isto mais presente nas instituições públicas de ensino que possuem pósgraduação. Todavia, tanto o ensino quanto a pesquisa ocupam um papel central na construção da identidade profissional do docente de nível superior (e.g., Martins, 2009; Nunes, 2012). O segundo eixo é o do vínculo institucional. Neste caso, o que está em jogo é a imagem e o suporte institucional dado às atividades centrais do docente, além daquelas que configuram qualquer vínculo empregatício como estabilidade, ascensão de carreira, etc. A escala de contrato psicológico de docentes apresentada neste artigo leva em conta que tal contrato está sustentando no modelo de trocas homomórficas e heteromórficas. Ao se levar em conta os dois eixos de vínculo profissional, o contrato psicológico docente estaria estruturado conforme descrito na Tabela 1.

Tabela 1 Estrutura do contrato psicológico docente

\begin{tabular}{c|l|l}
\hline & \multicolumn{1}{|c|}{ Homomórfica } & \multicolumn{1}{|c}{ Heteromórfica } \\
\hline \multirow{5}{*}{$\begin{array}{c}\text { Trocas } \\
\text { Acadêmicas }\end{array}$} & $\begin{array}{l}\text { Por dedicar-se a atividades que } \\
\text { asseguram a qualidade do ensino e da } \\
\text { pesquisa realizada, o docente espera } \\
\text { que a organização ofereça condiçães e } \\
\text { suporte que permitam esta dedicação e } \\
\text { investimento. }\end{array}$ & $\begin{array}{l}\text { Por dedicar-se a atividades que } \\
\text { asseguram a qualidade do ensino } \\
\text { e da pesquisa realizada, o docente } \\
\text { espera que a organização ofereça } \\
\text { outras formas de compensação. }\end{array}$ \\
\hline \multirow{5}{*}{$\begin{array}{l}\text { Trocas } \\
\text { Institucionais }\end{array}$} & $\begin{array}{l}\text { Para poder aderir e fidelizar-se aos } \\
\text { regulamentos e normas institucionais, } \\
\text { o docente espera que a organização } \\
\text { apresente de modo claro e seja } \\
\text { transparente em relação esta } \\
\text { normativa. }\end{array}$ & $\begin{array}{l}\text { Para poder aderir e fidelizar-se } \\
\text { aos regulamentos e normas } \\
\text { institucionais, o docente espera } \\
\text { que a organização ofereça outras } \\
\text { formas de compensação, como } \\
\text { estabilidade, respeito mútuo etc }\end{array}$ \\
\hline
\end{tabular}

Nota: Elaboração dos autores.

A compreensão da estrutura dos contratos psicológicos de docentes pode auxiliar no planejamento de ações de gestão no contexto de instituições educacionais públicas e privadas, 
assegurando o suporte necessário para o desempenho e desenvolvimento docente com qualidade de vida no trabalho. O presente artigo apresenta uma medida para subsidiar o diagnóstico e acompanhamento de contratos psicológicos de docentes de ensino superior com base na teoria da reciprocidade homomórfica e heteromórfica. A estrutura da medida foi concebida a partir dos dois eixos principais do trabalho do docente de ensino superior (ensinoaprendizagem e produção acadêmica/ pesquisa), além do vínculo institucional de trabalhador.

\section{MÉTODO}

\subsection{PARTICIPANTES}

Participaram 232 docentes que atuam no ensino superior de instituições públicas, privadas e confessionais. A amostra foi composta fazendo uso da técnica de snowball (Hancock \& Gile, 2011) a partir de contatos iniciais com docentes de todo o Brasil. Houve a prevalência de docentes do sexo feminino (57,5\%), com título de mestre $(48,2 \%)$, da região nordeste $(59,1 \%)$ e na faixa etária entre 30 e 49 anos $(80,4 \%)$, com uma média de idade média de 42 anos (dp=9,89). 67,4\% docentes atuavam na rede particular de ensino $(51,3 \%$ em instituições laicas e 16,1\% em confessionais), 32,6\% na rede pública. O tempo de experiência variou entre 1 e 34 anos $\left(x^{-}=7,5, d p=7,35\right)$, com a maior parte dos docentes tendo até 4 anos de experiência $(45,1 \%)$, sendo que a segunda faixa mais prevalente é a que vai de 5 a 8 anos $(25,4 \%)$, juntas perfazendo $70,5 \%$. Sobre a formação docente, $14 \%$ têm apenas a especialização, $48,2 \%$ o mestrado, $28 \%$ o doutorado e $9,8 \%$ o pós-doutorado.

\subsection{INSTRUMENTO}

A escala de contrato psicológico docente foi construída fazendo uso de técnicas qualitativas e quantitativas. Sustentada nas percepções de obrigações mútuas entre docentes e empregadores, e também nas regras de reciprocidade homomórficas e heteromórficas, a medida foi construída para abranger a maneira como os docentes percebem tanto as próprias obrigações, quanto as contrapartidas que as Instituições de Ensino Superior oferecem. Dada a inexistência de escalas para servirem de parâmetro para construção da medida foram realizadas entrevistas com docentes e coordenadores de curso, com o intuito de explorar qualitativamente as expectativas mútuas. Tendo em vista que a imprecisão das medidas de contrato sobre quem seria de fato a figura do empregador, para este estudo elegeu-se os coordenadores como representantes da instituição. A opção foi feita pelo fato de os 
coordenadores, além de docentes, exercerem função de gestão acadêmica, ocupando uma posição intermediária na escala decisória das políticas institucionais. Os resultados do estudo de Amparo Sobrinho (2011) com docentes brasileiros utilizando a medida de contrato psicológico acadêmico de Krivokapic-Skoko e O’Neill (2008) também foram consultados para a elaboração de alguns dos itens.

A escala ficou composta por 35 itens (15 sobre obrigações do docente e 20 sobre contrapartidas ou trocas institucionais), além de dois itens adicionais para avaliar o estado dos contratos, que se refere à percepção do quanto o contrato é cumprido. Os itens de obrigações docentes estão distribuídos em duas dimensões: a) acadêmica ( 8 itens, sendo 4 voltados para a pesquisa e produção acadêmica e 4 para o ensino) e b) institucional ( 7 itens). A primeira dimensão une elementos da atividade de ensino e de pesquisa. A segunda dimensão inclui itens que exploram o docente como empregado, envolvendo as suas obrigações para com a instituição, o trabalho em equipe, respeito às normas e afins. Além disso, para cada dimensão foram elaboradas contrapartidas (trocas) de natureza homomórfica (8 itens de contrapartida acadêmica e 7 itens de contrapartida institucional) e heteromórfica (5 itens). As trocas heteromórficas compunham um único bloco de itens que serviam tanto para avaliar as trocas heteromórficas das obrigações acadêmicas quanto obrigações institucionais do docente. A escala de resposta em todos os casos era tipo likert de sete pontos, em que quanto mais elevado o número, mais forte a percepção de ser uma obrigação do docente para com a organização (acadêmica e institucional) e obrigação da organização para com o docente (acadêmica homomórfica, institucional homomórfica, trocas heteromórficas)

\section{PROCEDIMENTOS DE VALIDAÇÃO E ANÁLISE DE DADOS}

$\mathrm{Na}$ etapa da análise semântica da escala solicitou-se a 15 docentes que atuassem como juízes e avaliassem a clareza dos itens e sua adequação a cada uma das dimensões propostas. Os docentes eram de diferentes áreas, com mais de 10 anos de experiência. Com base nas avaliações, quatro itens foram ajustados para uma melhor adequação à dimensão para qual foram concebidos. Feito isso, foi conduzido um pré-teste com mais 10 docentes do ensino superior, para avaliar o nível de compreensão dos itens e das respostas na versão final, não sendo apontada a necessidade de ajustes.

Em termos dos procedimentos para as análises de dados foram utilizados os softwares SPSS 21 e AMOS 18. Inicialmente foi observada a adequação quanto ao tamanho da amostra, além de eventuais dados omissos, tendo sido eliminados 24 casos. Em seguida, foram 
avaliadas possíveis violações à normalidade, multicolinearidade e casos extremos multi e univariados. Os valores de assimetria e curtose variaram, respectivamente, entre $|-1,925|$ e $\mid-$ 0,043|, e entre $|-1,228|$ e $|4,079|$, mostrando-se adequados em relação aos critérios apresentados em Kline (2011; $\mid$ curtose $\mid<10$ e $\mid$ assimetria $\mid<3)$. Foram identificados 8 casos extremos a partir da distância de Mahalanobis, tendo sido excluídos da base. Foram ainda feiras análises do índice de fatorabilidade KMO e do teste de esfericidade de Bartlett, que indicaram a adequação da amostra à análise fatorial. Procedeu-se também à análise de confiabilidade para avaliar a consistência interna, tendo sido eliminados dois itens: um das obrigações acadêmicas e outro das contrapartidas institucionais. Desse modo, a escala final ficou com 33 itens. Por fim, foi feita a análise fatorial confirmatória por meio de modelagem de equações estruturais, com base no método da Máxima Verossimilhança. Para fins de teste do modelo de medida foram formadas parcelas a partir dos fatores (Little et al., 2002), de sorte que o modelo fosse composto de sete parcelas para obrigações do docente e sete para as contrapartidas institucionais. As parcelas das obrigações do docente eram divididas em quatro relacionadas à dimensão acadêmica (duas vinculadas ao ensino e duas vinculadas à pesquisa $\mathrm{e}$ produção acadêmica), e três relacionadas ao papel do docente como empregado. As parcelas relacionadas às contrapartidas institucionais se dividiram em cinco parcelas homomórficas (das quais três eram na esfera acadêmica e duas na esfera institucional) e duas parcelas heteromórficas. Para avaliar o ajuste do modelo, foram considerados os índices $\left(\chi^{2}-\right.$ quiquadrado de ajustamento; $\chi^{2} / \mathrm{gl}$ - razão entre qui-quadrado e graus de liberdade; TLI - índice de Tucker-Lewis; CFI - índice de ajuste comparativo; AIC - critério de informação de Akaike; e RMSEA - raiz do erro quadrático médio de aproximação) onde o modelo teorizado (ou modelo proposto) foi constrastado com um modelo alternativo para verificar qual deles se ajustava melhor aos dados e explicava a estrutura da medida.

No caso da escala de obrigações do docente, o modelo alternativo considerava todas as variáveis observadas compondo uma única variável latente de obrigações docentes (modelo unifatorial). O modelo teorizado, por sua vez, dividia as obrigações do docente em obrigações acadêmicas (subdivididas em ensino e pesquisa) e obrigações institucionais (relacionadas ao papel do docente enquanto empregado da instituição).

Para a escala de contrapartidas da IES, o modelo alternativo considerou as trocas heteromórficas como uma faceta e as homomórficas como outra (independentemente de serem de natureza acadêmica ou institucional). O modelo proposto (conforme visto na Figura 
2 mais adiante) considera três facetas: (1) as trocas homomórficas de natureza acadêmica; (2) as trocas homomórficas de natureza institucional; e (3) as trocas heteromórficas

\section{RESULTADOS}

A Tabela 2 apresenta os índices globais de ajuste dos modelos conceituais de ambas as escalas: obrigações do docente e contrapartidas institucionais. Conforme se vê na Tabela 2, o modelo proposto (que discrimina obrigações acadêmicas e institucionais) apresenta melhores índices de ajustes $(\chi 2=16,170, \mathrm{CFI}=0,993$, TLI $=0,988$ e RMSEA $=0,042)$, que o modelo alternativo $(\chi 2=419,868, \mathrm{CFI}=0,442, \mathrm{TLI}=0,164$ e $\mathrm{RMSEA}=0,375)$, o que também pode ser observado na redução significativa do valor de AIC.

Tabela 2.Índices de Ajuste dos Modelos Testados para ambas as escalas

\begin{tabular}{lcccc}
\hline & \multicolumn{2}{c}{ Escala de Obrigações do Docente } & \multicolumn{2}{c}{ Escala de Obrigações da Instituição } \\
\hline \multicolumn{1}{c}{ Modelo } & Proposto & Alternativo & Proposto & Alternativo \\
\hline$\chi^{2}$ & 16,170 & 419,868 & 12,338 & 121,820 \\
$\mathrm{gl}$ & 12 & 14 & 11 & 19 \\
$\chi^{2} / \mathrm{gl}$ & 1,347 & 29,991 & 1,122 & 6,412 \\
TLI & 0,988 & 0,164 & 0,993 & 0,773 \\
CFI & 0,993 & 0,442 & 0,996 & 0,846 \\
AIC & 62,17 & 461,868 & 60,338 & 171,820 \\
RMSEA & 0,042 & 0,375 & 0,025 & 0,162 \\
\hline
\end{tabular}

Nota: Parâmetros estimados com $p<0,001$. Os valores de referência (Byrne, 2010; Marôco, 2010) foram: TLI e $\mathrm{CFI} \geq 0,90 ; \mathrm{RMSEA} \leq 0,05 ; \chi^{2} / \mathrm{gl} \sim 1 ; \chi^{2}=$ quanto menor, melhor. $\mathrm{AIC}=$ quanto menor, melhor.

Com base em consulta aos índices de modificação incluiu-se uma trajetória entre os erros 3 e 4 da dimensão acadêmica (itens sobre ensino), obtendo-se melhoria adicional dos indicadores. 


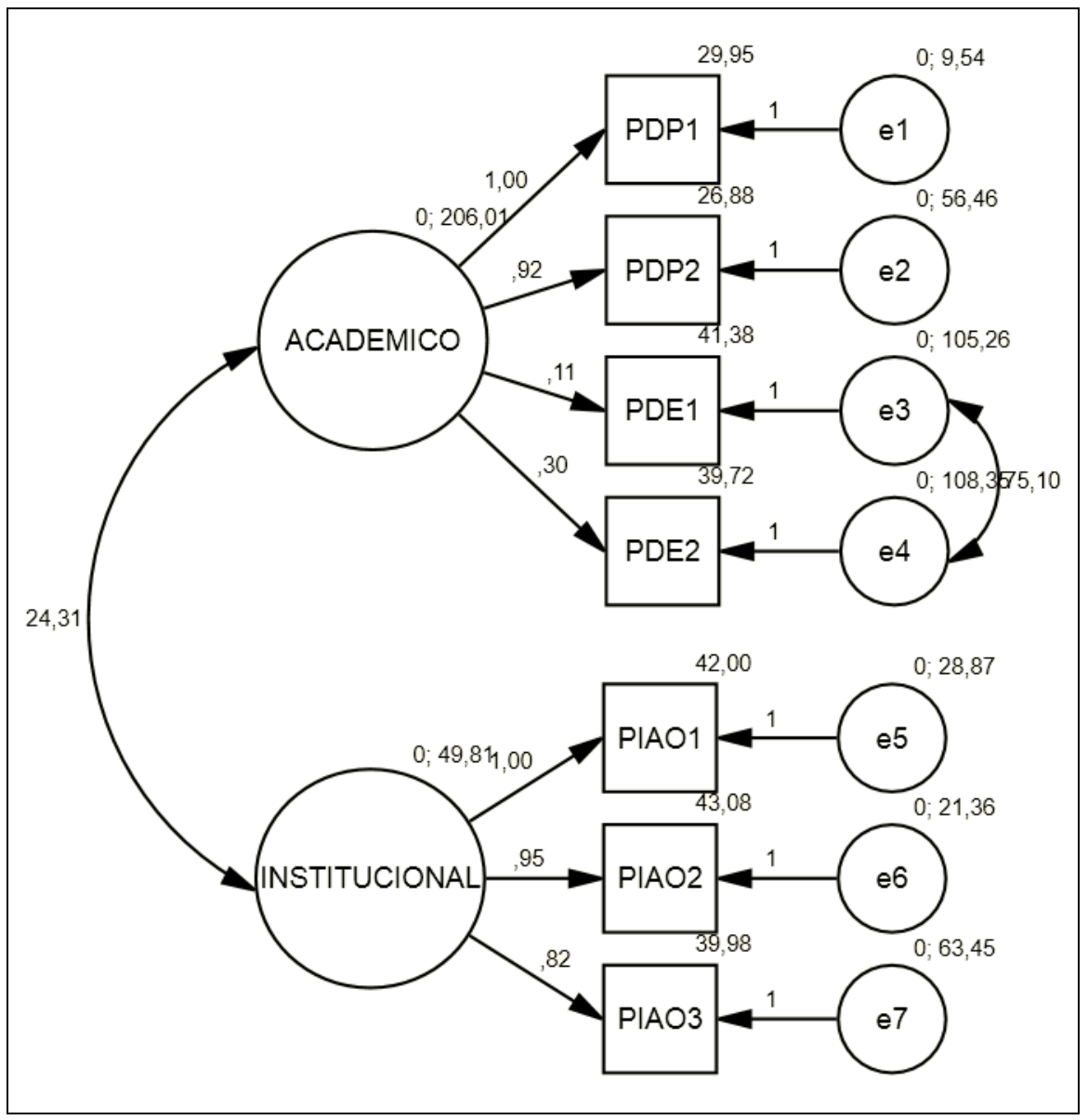

Figura 1 Modelo da estrutura da Escala de Obrigações do Docente.

Conforme dito na seção de método, em relação à Escala de Contrapartidas Institucionais, foram avaliados também dois modelos: um que considerava as contrapartidas homomórfica e contrapartidas heteromórficas (modelo alternativo); e o modelo proposto, que considerava três facetas (contrapartidas homomórficas acadêmicas; contrapartidas homomórficas institucionais; e contrapartidas heteromórficas). Os índices de ajuste observados na Tabela 2 para a Escala de Contrapartidas Institucionais indicam um ajuste pobre do modelo alternativo unifatorial $(\chi 2=121,820, \quad \mathrm{CFI}=0,846, \quad \mathrm{TLI}=0,773 \quad \mathrm{e}$ RMSEA $=0,162)$, e um bom ajuste do modelo proposto para a ferramenta $(\chi 2=12,338$, $\mathrm{CFI}=0,996$, TLI=0,993 e RMSEA=0,025), além da redução do valor de AIC, passando de 171,820 para 60,338 , o que representa uma melhora do modelo. 


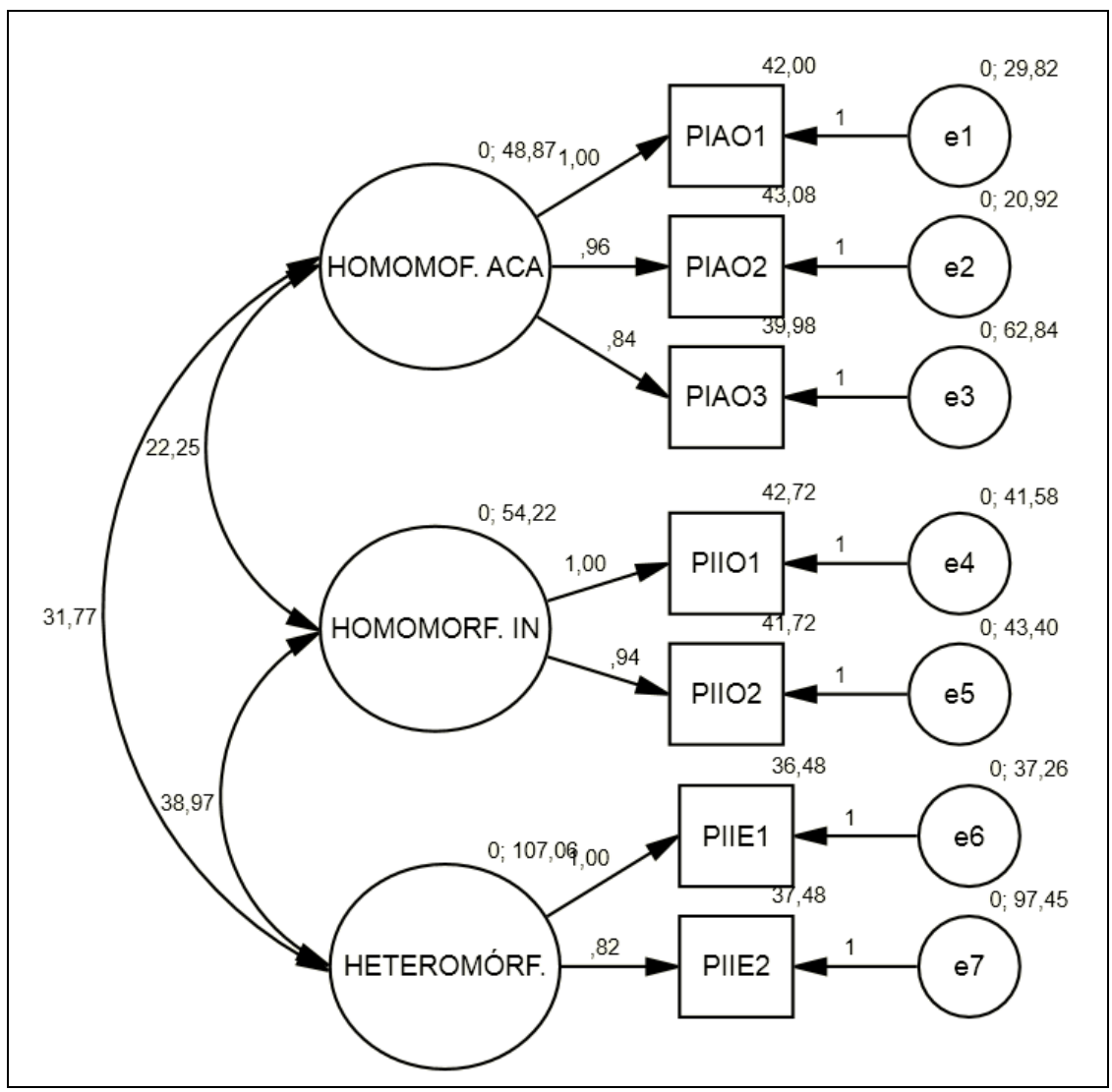

Figura 2 Modelo da estrutura da Escala de Contrapartidas da IES.

A tabela 3 apresenta a estrutura fatorial da escala de contratos psicológicos docentes com os indicadores que permitem demonstrar a confiabilidade da medida.

Tabela 3 Confiabilidade - Consistência interna

\begin{tabular}{lccc}
\hline & & $\begin{array}{c}\text { Coeficiente de alfa } \\
\text { de Cronbach }\end{array}$ & $\begin{array}{c}\mathrm{n}^{\text {o de }} \\
\text { Itens }\end{array}$ \\
\hline \multirow{3}{*}{ Obrigações Docentes } & Ensino & 0,93 & 4 \\
& Pesquisa & 0,80 & 4 \\
& Institucional & 0,83 & 6 \\
Contrapartidas Institucionais & Homomórficas Acadêmicas & 0,86 & 6 \\
& Homomórficas Institucionais & 0,78 & 5 \\
& Heteromórficas & 0,78 & 5 \\
\hline
\end{tabular}

Nota: O valor de referência (Corrar 2007; Hair et al., 2010; Pasquali, 2003) foi alfa $\geq 0,7$

Os contratos psicológicos dos docentes da amostra podem ser assim descritos. As obrigações acadêmicas de ensino $\left(\mathrm{x}^{-}=6,26, \mathrm{dp}=1,03\right)$ e as obrigações institucionais $\left(\mathrm{x}^{-}=\right.$ $6,02, \mathrm{dp}=0,80)$ apresentaram maiores médias que as relacionadas à pesquisa $\left(\mathrm{x}^{-}=5,12\right.$, $\mathrm{dp}=1,63) \quad(\mathrm{F}=74,995 ; \mathrm{p}<0,001) . \quad$ Para elevados investimentos, os docentes esperam contrapartidas substanciais das instituições de ensino superior. As contrapartidas homomórficas tanto acadêmicas $\left(\mathrm{x}^{-}=6,33, \mathrm{dp}=0,90\right)$ quanto institucionais $\left(\mathrm{x}^{-}=6,43\right.$, 
$\mathrm{dp}=0,73)$ são mais esperadas que as trocas heteromórficas acadêmicas $\left(\mathrm{x}^{-}=5,73, \mathrm{dp}=1,35 \mathrm{e}\right.$ institucionais $\left(\mathrm{x}^{-}=5,96, \mathrm{dp}=1,02\right)(\mathrm{F}=35,525 ; \mathrm{p}<0,001)$.

\section{DISCUSSÃO}

O presente estudo teve o objetivo de construir e validar uma medida de contratos psicológicos de docentes do ensino superior brasileiro. Os índices de ajuste do modelo foram consistentes com a expectativa conceitual de que o contrato psicológico de docentes de nível superior assenta-se na relação entre obrigações docentes (acadêmicas e institucionais) e trocas homomórficas e heteromórficas (Chiuzi, 2014; Gouldner, 1960). Os resultados sugerem que os docentes de ensino superior percebem-se mais obrigados a desempenharem tarefas relacionadas ao ensino que a pesquisa, mas se percebem também obrigados a sustentarem um vínculo com a instituição empregadora como qualquer outro trabalhador. A ênfase nas obrigações de ensino pode ser justificativa pelo fato de a maior parte das instituições privadas investirem menos em pesquisa, sendo a experiência de ensino a prevalente neste contexto. Outra explicação pode ser a de as atividades de ensino estarem no centro da identidade profissional de docentes. Supõe-se que a identidade profissional com a docência pode fazer com que prevaleça a expectativa de trocas homomórficas tanto em relação a atividades-fim quanto atividades-meio, que sustentariam tanto a motivação acadêmica quanto o vínculo institucional. Os resultados sinalizam também que as trocas homomórficas também são esperadas quando o docente pensa no investimento que ele faz na instituição educacional empregadora. Ao servir como ferramenta de diagnóstico pode vir a ajudar na gestão de contratos psicológicos (Menegon \& Casado, 2006) de docentes, direcionando de maneira mais clara as ações nesse contexto.

Apesar dos resultados positivos obtidos no estudo, algumas considerações merecem ser feitas do ponto de vista de sua análise crítica, limitações e recomendações para o futuro. Em primeiro lugar, a amostra utilizada não é representativa dos docentes de ensino superior no Brasil. Houve sub-representação dos docentes de instituições particulares e confessionais, e super-representação dos docentes do ensino público (INEP, 2014; Martins, 2009). Tendo em vista as especificidades desses contextos, sobretudo no que se refere ao investimento em pesquisa e extensão, bem como na segurança do vínculo, é natural que haja diferenças na forma como os contratos psicológicos se estruturam. Dessa maneira, por um lado, é importante a condução de estudos que possam representar o perfil dos docentes do país de maneira mais representativa e, por outro lado, a condução de estudos que avaliem 
comparativamente a maneira como o construto se apresenta nos dois contextos: público e privado. Essa necessidade se evidencia, tendo em conta as diferentes políticas de trabalho adotadas para o docente, além das diferenças na orientação desses dois tipos de instituição educacional

\section{REFERÊNCIAS}

AMPARO SOBRINHO, J.B. Contrato psicológicos de professores de ensino superior de instituições públicas e particulares. Dissertação de mestrado em psicologia. UFBA - PPGP, Salvador, 2011.

BAL, P.M., \& SMIT, P. The older the better!: Age-related differences in emotion regulation after psychological contract breach. Career Development International. 17(1), 6-24, 2012.

BAL, P.M., CHIABURU, D.S., \& DIAZ, I. Does psychological contract breach decrease proactive behaviors? The moderating effect of emotion regulation. Group \& Organization Management, 36(6), 722-758, 2011.

BAO, Y., OLSO, B., PARAYITAM, S., \& ZHAO, S. The effects of psychological contract violation on Chinese executives. The International Journal of Human Resource Management, Vol 22(16), 2011.

BASTOS, A.V.B., MAIA, L.G., RODRIGUES, A.C.A., MACAMBIRA, M.O., \& BORGESANDRADE, J.E.Vínculos dos Indivíduos com a Organização: Análise da Produção Científica Brasileira 2000-2010. Psicologia: Teoria e Pesquisa, Abr-Jun, Vol. 30 n. 2, pp. 153-162, 2014.

BORDIA, P., RESTUBOG, S.L.D., \& TANG, R.L.When employees strike back: investigating mediating mechanisms between psychological contract breach and workplace deviance. Journal of Applied Psychology, Vol 93(5), Sep, p.1104-1117, 2008.

BYRNE, B.M. Structural equation modelong with AMOS: Basic concepts, applications, and programming. New York, NY: Routledge, Taylor \& Francis, 2010.

CARVALHO, C.H.A. Política para o ensino superior no Brasil (1995-2008). Ruptura e continuidade nas relações entre público e privado. In: J. R. S., SILVA, J. F. OLIVEIRA \& D. MANCEBO. (Orgs.) Reforma universitária: dimensões e perspectivas. Campinas: Alínea, p. 125-139, 2006.

CAVANAUGH, M., \& NOE, R. Antecedents and consequences of relational components of the new psychological contract. Journal of Organizational Behavior, Vol. 20 (3), 323$340,1999$.

CHIN, P.L., \& HUNG, M.L. Psychological contract breach and turnover intention: the moderating roles of adversity quotient and gender. Social Behavior and Personality. Vol. 41, No.5 pp843-860, 2013. 
CHIUZI, R.M., \& MALVEZZI, S. Mais de 50 anos de pesquisa sobre contratos psicológicos de trabalho: sistematização teórica e sugestão de agenda futura de pesquisa. Revista de Carreiras e Pessoas São Paulo. Volume IV - Número 03 - Set/Out/Nov/Dez 2014, pp. $282-294,2014$.

CHIUZI, R.M. As dinâmicas de formação e continuidade dos contratos psicológicos de trabalho. (Tese de doutorado, Instituto de Psicologia, USP), 2014.

CHU, H., \& KUO, T. Exploring Faculty Psychological Contract through leadership style and institutional climate in a higher education setting. International Journal of Business and Social Science. Vol. 3, No.4 pp159-164, 2012.

CONWAY, N., \& BRINER, R.B. A daily diary study of affective responses to psychological contract breach and exceeded promises. Journal of Organizational Behavior, Vol. 23, 287-302, 2002.

COOPER, C.L. A natureza mutante do trabalho: o novo contrato psicológico e os estressores associados. In A.M. Rossi, P.L. Perrewé, \& S. L. Sauter (Orgs.) Stress e Qualidade de Vida no Trabalho (pp.3-8). São Paulo: Atlas, 2008.

CORRAR, L.J., PAULO, E., \& FILHO, J.M.D. (ORGS.). Análise Multivariada. São Paulo: Atlas, 2007.

DABOS, G. E., \& ROUSSEAU, D. M. Mutuality and reciprocity in the psychological contracts of employees and employers, Journal of Applied Psychology, 89 (1), 52-72, 2004.

FARNSWORTH, E.A. Contracts. Boston: Little Brown, 1982.

FRANCO, A. P. Ensino Superior no Brasil: cenário, avanços e contradições. Jornal de Políticas educacionais, 4, 53-63, 2008.

GAMMIE, R. P. Psychological Contracts in a Business School Context. (Tese de doutorado) University Of Starling, 2006.

GOULDNER, A. W. The norm of reciprocity: A preliminary statement. American Sociological Review, 25, 161-178, 1960.

GUEST, D., \& CONWAY, N. Communicating the psychological contract: An employer perspective. Human Resource Management Journal,12, 22-38, 2002.

HAIR, J.F., BLACK, W.C., BABIN, B.J., ANDERSON, R.E., \& TATHAM, R.L. Análise Multivariada de dados. Porto Alegre: Bookman, 2010.

HAO ZHAO, WAYNE, S.J., GLIBKOWSKI, B.C., \& BRAVO, J. The impact of psychological contract breach on work-related outcomes: a meta-analysis. Personnel Psychology, 60, 640-680, 2007. 
HERRIOT, P., MANNING, W. E. G., \& KIDD, J. M. The content of the psychological contract. British Journal of Management, 8, 151-162, 1997.

INEP - Instituto Nacional de Estudos e Pesquisas Educacionais Anísio Teixeira. Censo da educação superior, Brasilia, 2014. Recuperado em 23/04/2015 em

http://portal.inep.gov.br/web/censo-da-educacao-superior

JAMIL, A.; RAJA, U., \& DARR, W. Psychological Contract Types as Moderator in the Breach-Violation and Violation-Burnout Relationships. The Journal of Psychology, 147(5), 491-515, 2013.

JANSSENS, M.; SELS, L., \& VAN DEN BRANDE, I. Multiple types of psychological contracts - a six-cluster solution. Human Relations, 56 (11). 1349-1378, 2003.

KICKUL, J. When organizations break their promises: Employee reactions to unfair processes and treatment. Journal of Business Ethics, 29, 289-307, 2001.

KLINE, R.B. Principles and practice of structural equation modelling. New York, NY: Guilford Press, 2011.

KRIVOKAPIC-SKOKO, B., \& O’NEILL, G. University Academics' Psychological Contracts in Australia: a Mixed Method Research Approach. The Electronic Journal of Business Research Methods, 6 (1) 61-72, 2008.

LAPALME, M.È.; SIMARD, G., \& TREMBLAY, M. The influence of psychological contract breach on temporary workers' commitment and behaviors: A multiple agency perspective. Journal of Business and Psychology, Vol 26(3), 311-324, 2011.

LAUS, S., \& MOROSINI, M.C. Internacionalización de la educación superior en Brasil, Colombia: Banco Mundial en coedición con Mayol Ediciones, 2005.

LITTLE, T. D., CUNNINGHAM, W. A., SHAHAR, G., \& WIDAMAN, K. F. To parcel or not to parcel: Exploring the question, weighing the merits. Structural Equation Modeling, 9, 151-173, 2002.

MARÔCO, J. Análise de equações estruturais. Pêro Pinheiro, Portugal: Report Number, 2010.

MARTINS, C.B. A reforma universitária de 1968 e a abertura para o ensino superior privado no Brasil. Educ. Soc., Campinas, vol. 30, n.106, p.15-35, jan./abr, 2009.

MENEGON, L.F., \& CASADO, T. O contrato psicológico como ferramenta para a gestão de pessoas. Revista de Administração., São Paulo, 41 (2), 125-135, 2006.

NUNES, E.O. Educação superior no Brasil: estudos, debates e controvérsias. Rio de Janeiro: Garamond, 2012.

PASQUALI, L. Psicometria: Teoria dos testes na Psicologia e na Educação. Rio de Janeiro: Vozes, 2003. 
RIOS, M. C., \& GONDIM, S. M. G. Contrato psicológico de trabalho e a produção acadêmica no Brasil. Revista Psicologia: Organizações e Trabalho, 10(1), 23-363 2010.

RIOS, M.C., \& GONDIM, S.M.G. Contratos Psicológicos e Terceirização: Um estudo das relações entre vínculos e as práticas de gestão de pessoas, Organizações \& Sociedade vol.17 no.55, 2010.

ROBINSON, S. L., \& MORRISON, E. W. The development of psychological contract breach and violation: a longitudinal study. Journal of Organizational Behavior, 21, 525$546,2000$.

ROUSSEAU, D.M. (1995). Psychological contracts in organizations - understanding written and unwritten agreements Thousand Oaks, CA: Sage.

SEVERINO, A.J. O ensino superior brasileiro: novas configurações e velhos desafios. Educar, Curitiba, n. 31, p. 73-89, Editora UFPR, 2008.

SHEN, J. University academics' psychological contracts and their fulfillment. Journal of Management Development, 29 (6), 575-591, 2010.

STALliVIERI, L. O Sistema de Ensino Superior do Brasil: Características, Tendências e Perspectivas. Educación superior en América Latina y el Caribe: Sus estudiantes hoy. México, 2007.

TIPPLES, R., KRIVOKAPIC-SKOKO, B., \& O’NEILL, G. University Academics' Psychological Contracts in Australia and New Zealand. New Zealand Journal of Employment Relations. 32(2): 32 - 52, 2007.

WADE-BENZONI, K., ROUSSEAU, D., \& LI, M. Managing relationships across generations of academics. International Journal of Conflict Management. 17(1): 4-33, 2006

WELLIN, M. Managing the Psychological Contract: Using the personal deal to increase business performance. Cornwall: MPG Books, 2007. 